\title{
Factores que influyen en el diagnóstico normal por antropometría de niños de 0 a 3 años del AAHH Nueva Esperanza, Nuevo Chimbote, 2011
}

\author{
Yenny Sandra TANTARICO FACHO*
}

EAP Nutrición, Facultad de Ciencias de la Salud, Universidad Peruana Unión, Alt. Km 19; Carretera Central, Lurigancho, Lima

\begin{abstract}
RESUMEN
El objetivo de esta investigación fue identificar los factores que influyen en el diagnóstico nutricional normal de los niños y niñas de 0 a 3 años del área de estudio. Se aplicó un barrido de peso y talla a los niños de Nueva Esperanza para identificar el diagnóstico nutricional normal, luego se aplicó la encuesta de cuidado en el hogar y la encuesta de salud y nutrición, a las madres con niños menores de 36 meses. Los resultados muestran que los factores de nutrición, de salud y de cuidado en la familia influyen significativamente en un nivel de significancia del $95 \%$ en el diagnóstico nutricional normal de niños de 0 a 3 años del AAHH Nueva Esperanza, de Nuevo Chimbote.
\end{abstract}

Palabras clave: Diagnóstico nutricional, factores nutricionales, factores de salud, niños.

\begin{abstract}
Ojective: The objective of this research was to identify the factors that influence in the normal nutritional diagnosis of boys and girls from 0 to 3 years old of the area of study. Materials y methods: We applied a weight and height swept to the Nueva Esperanza's kids in order to identify normal nutritional diagnosis, then we applied a survey about house care, health and nutrition to mothers with kids under 36 months old. Results: The results show that the nutritional, health and family care factors influence in a significant way in a level of significance of $95 \%$ in the normal nutritional diagnosis of kids from 0 to 3 years old of the AAHH Nueva Esperanza, in Nuevo Chimbote.
\end{abstract}

Keywords: Nutritional diagnosis, nutritional factors, health factors, children.

* Correspondencia: yennytantarico@hotmail.com 


\section{INTRODUCCIÓN}

Los niños y niñas nutricionalmente sanos, indican el adelanto o progreso de un país; siendo que la familia es una institución fundamental para su desarrollo. Uno de los problemas graves que todavía enfrenta nuestro país es la desnutrición; sin embargo, existe un porcentaje de niños que no tiene desnutrición, nos interesa conocer y dar a conocer qué hacen las madres de esos niños y niñas sanos, siendo que tienen similares oportunidades que las madres que tienen a sus niños desnutridos.

"Un buen estado nutricional ayuda al buen desarrollo físico e intelectual, y contribuye a asegurar una vida larga y saludable". (Behrman, 2000). Identificando el estado nutricional de una comunidad y sus factores, permite evaluar los programas de salud para poder hacer cambios necesarios. La nutrición tiene durante su período prenatal y en los primeros años de vida gran importancia por el efecto que ejerce sobre el desarrollo normal del niño.

El Plan Nacional de Acción por la infancia y la adolescencia (2002- 2010), menciona el interés del estado peruano en mejorar las condiciones de vida de los niños, donde se establecieron cuatro principios rectores: La igualdad de oportunidades para todos los niños; el fortalecimiento del desarrollo del niño como sujeto de derechos y como principal sustento del desarrollo del niño y su derecho a participar y el reconocimiento de la familia como institución fundamental para el desarrollo del ser humano.

El crecimiento es un proceso continuo desde la concepción hasta la edad adulta, determinado por la carga genética de cada individuo y dependiente, tanto de factores ambientales como de un correcto funcionamiento del sistema neuroendocrino. Su futuro depende, en gran medida, del conocimiento del mismo y de la vigilancia no solo del ser humano sino de la población a la cual pertenece. La forma más fácil, económica y universalmente aplicable para observar el crecimiento físico es la antropometría; del mismo modo la auxología es uno de los pilares de la ciencia que estudia el crecimiento y desarrollo del ser humano. (Delgado, 1986).

La antropometría permite conocer el patrón de crecimiento propio de cada individuo, evaluar su estado de salud y nutrición, detectar alteraciones, predecir su desempeño, su salud y sus posibilidades de supervivencia (Behrman, 2000). En el ámbito de poblaciones constituye un elemento valioso para la toma de decisiones en cuestiones de salud pública; su utilidad radica en que las medidas antropométricas son un indicador del estado de las reservas proteicas y de tejido graso del organismo. Se emplea tanto en niños como en adultos (OMS, 1995).

Los indicadores antropométricos permiten evaluar a los individuos directamente y comparar sus mediciones con un patrón de referencia generalmente aceptado a nivel internacional y así identificar el estado de nutrición, diferenciando a los individuos nutricionalmente sanos de los desnutridos, obesos y personas con sobrepeso (OMS, 1995).

Los indicadores antropométricos más comunes que conocemos son: a) peso-talla, b) talla-edad, c) peso-edad. El objetivo del presente estudio es identificar los factores que influyen en el diagnóstico nutricional normal por antropometría de los niños y niñas de 0 a 3 años del área de estudio

\section{MATERIAL Y MÉTODOS}

Estudio explicativo descriptivo, diseño correccional causal. La intervención fue realizada a 52 madres con niños menores de 36 meses del AAHH Nueva Esperanza del Programa Vaso de leche. Niños nacidos en marzo del 2009 a diciembre del año 2011, la mayoría son inmigrantes de las zonas rurales de la Sierra (Huaraz, sierra de Piura, de la Selva (San Martín, Amazonas) y de la Costa (Ica, Chiclayo).

Los participantes del estudio viven en viviendas construidas precariamente con esteras y triplays, ubicadas en los arenales de Nuevo Chimbote, se caracterizan por su extrema pobreza, donde se practica una economía de subsistencia y dificultades de accesibilidad económica a los servicios básicos de salud, vivienda y educación. El proceso de la intervención del trabajo duró 12 semanas, iniciándose con el pesado y tallado de los niños para ver su diagnóstico nutricional y posteriormente aplicar la encuesta casa por casa de cada participante en coordinación con el personal profesional del programa vaso de leche.

Se aplicó el cuestionario de línea de base del DAP 2002-2007 elaborado por ADRA Perú que consta de 7 secciones: Características Demográficas, nutrición, monitoreo del crecimiento, diarrea, cuidado materno, inmunización, agua y saneamiento.

Para el análisis de datos se utilizó el software SPSS 19 y el estadístico utilizado fue la prueba de t de student $95 \%$ de confianza, para identificar qué factores han influenciado en el diagnóstico nutricional normal de niñas y niños menores de 3 años. 


\section{RESULTADOS}

Tabla 1

Proporción de niños menores de tres años con desnutrición

\begin{tabular}{|c|c|c|c|c|c|c|c|}
\hline & & \multicolumn{6}{|c|}{ SEXO DEL NIÑO (A) } \\
\hline \multicolumn{2}{|c|}{ Tipos de desnutrición } & \multicolumn{2}{|c|}{ Masculino } & \multicolumn{2}{|c|}{ Femenino } & \multicolumn{2}{|c|}{ Total } \\
\hline & & $\mathrm{N}$ & $\%$ & $\mathrm{~N}$ & $\%$ & $\mathrm{~N}$ & $\%$ \\
\hline \multirow[t]{2}{*}{$\begin{array}{l}\text { Desnutrición } \\
\text { crónica }\end{array}$} & $\begin{array}{c}\text {-2 Desnutrición } \\
\text { crónica }\end{array}$ & 1 & 3.6 & 2 & 8 & 3 & 5.6 \\
\hline & $\begin{array}{c}-3 \text { Desnutrición } \\
\text { grave }\end{array}$ & 1 & 3.6 & - & - & 1 & 1.9 \\
\hline \multirow[t]{2}{*}{$\begin{array}{l}\text { Desnutrición } \\
\text { aguda }\end{array}$} & $\begin{array}{c}\text {-2 Desnutrición } \\
\text { aguda }\end{array}$ & 1 & 3.6 & - & - & 1 & 1.9 \\
\hline & $\begin{array}{c}\text {-3 Desnutrición } \\
\text { grave }\end{array}$ & - & - & - & - & - & - \\
\hline \multirow[t]{2}{*}{$\begin{array}{l}\text { Desnutrición } \\
\text { global }\end{array}$} & $\begin{array}{c}\text {-2 Desnutrición } \\
\text { global }\end{array}$ & 1 & 3.6 & - & - & 1 & 1.9 \\
\hline & $\begin{array}{c}-3 \text { Desnutrición } \\
\text { grave }\end{array}$ & - & - & - & - & - & - \\
\hline \multirow{2}{*}{$\begin{array}{c}\text { Niños con } \\
\text { diagnóstico } \\
\text { normal }\end{array}$} & 0 - -1.9 NORMAL & 24 & 85.7 & 23 & 92 & 47 & 88.7 \\
\hline & 0 - 1.9 NORMAL & - & - & - & & - & - \\
\hline Total & & 28 & 100 & 25 & 100 & 53 & $100 \%$ \\
\hline
\end{tabular}

En la tabla 1 se muestra que el 5.6\% de los niños tenían desnutrición crónica Por otro lado, el 1.9\% de los niños tuvo desnutrición aguda con menos dos desviaciones estándares. Siendo que la mayoría 88.7\% presentó un peos y talla adecuado para su edad.

\section{Tabla 2}

Prueba t student para medir la influencia de los Factores Nutricionales en el diagnóstico nutricional normal

\begin{tabular}{|c|c|c|c|c|c|c|}
\hline & \multirow[b]{2}{*}{$\mathrm{T}$} & \multirow[b]{2}{*}{ Gl } & \multirow{2}{*}{$\begin{array}{c}\text { Sig. } \\
\text { (Bilateral) }\end{array}$} & \multirow{2}{*}{$\begin{array}{l}\text { Diferencia } \\
\text { de Medias }\end{array}$} & \multicolumn{2}{|c|}{$\begin{array}{l}\text { 95\% Intervalo De Confianza } \\
\text { Para La Diferencia }\end{array}$} \\
\hline & & & & & Inferior & Superior \\
\hline $\begin{array}{c}\text { Niños con } \\
\text { diagnóstico normal }\end{array}$ & $-677,989$ & 46 & ,000 & $-48,872$ & $-49,02$ & $-48,73$ \\
\hline Lactancia materna & $-342,000$ & 6 & ,000 & $-48,857$ & $-49,21$ & $-48,51$ \\
\hline $\begin{array}{l}\text { Frecuencia de } \\
\text { Alimentación }\end{array}$ & $-142,793$ & 7 & ,000 & $-44,750$ & $-45,49$ & $-44,01$ \\
\hline Peso al Nacer & $-520,846$ & 46 & 000 & $-48,745$ & $-48,93$ & $-48,56$ \\
\hline
\end{tabular}

De acuerdo a la prueba t student, según se observa en la tabla 2, los factores nutricionales tales como la lactancia materna, frecuencia de alimentación y el peso al nacer influyen significativamente en el diagnóstico nutricional normal. Siendo la probabilidad Sig. (bilateral $=.000)$ es menor que alfa $(\alpha=0.05)$ a un nivel de significancia del $95 \%$. Por lo tanto, se rechaza la hipótesis nula y se acepta la hipótesis principal, concluyendo que los factores nutricionales influyen significativamente en el diagnóstico nutricional normal de niños de 0 a 3 años del AA.HH. Nueva Esperanza, de Nuevo Chimbote. 
Tabla 3

Prueba t student para medir la influencia de los factores de atención en salud en el diagnóstico nutricional normal

\begin{tabular}{|c|c|c|c|c|c|c|}
\hline & \multirow[b]{2}{*}{$\mathrm{t}$} & \multirow[b]{2}{*}{ gl } & \multirow{2}{*}{$\begin{array}{c}\text { Sig. } \\
\text { (bilateral) }\end{array}$} & \multirow{2}{*}{$\begin{array}{r}\text { Diferencia } \\
\text { de medias }\end{array}$} & \multicolumn{2}{|c|}{$\begin{array}{c}\text { 95\% Intervalo de } \\
\text { confianza para la diferencia }\end{array}$} \\
\hline & & & & & Inferior & Superior \\
\hline $\begin{array}{c}\text { NIÑOS CON DIAGNÓSTICO } \\
\text { NORMAL }\end{array}$ & $-677,989$ & 46 & ,000 & $-48,872$ & $-49,02$ & $-48,73$ \\
\hline Cobertura de inmunizaciones & $-302,035$ & 38 & 000 & $-48,103$ & $-48,42$ & $-47,78$ \\
\hline Prevalencia de diarrea & $-263,402$ & 16 & 000 & $-47,831$ & $-48,46$ & $-47,27$ \\
\hline Uso de saneamiento & $-589,449$ & 46 & 000 & $-46,191$ & $-46,35$ & $-46,03$ \\
\hline Eliminación de excretas & $-643,843$ & 46 & 000 & $-48,894$ & $-49,05$ & $-48,74$ \\
\hline Fuentes de agua para beber & $-280,017$ & 46 &, 000 & $-46,532$ & $-46,87$ & $-46,20$ \\
\hline
\end{tabular}

En la tabla 3, se observa que según la prueba t student, los factores de atención en salud tales como la cobertura de inmunizaciones, prevalencia de diarrea, uso de saneamiento, eliminación de excretas, fuentes de agua para beber influyen significativamente en el diagnóstico nutricional normal. Siendo la probabilidad Sig. (bilateral $=.000)$ es menor que alfa $(\alpha=0.05)$ a un nivel de significancia del 95\%. Por lo tanto, se rechaza la hipótesis nula y se acepta la hipótesis principal, concluyendo que los factores de atención en salud influyen significativamente en el diagnóstico nutricional normal de niños de 0 a 3 años del AA.HH. Nueva Esperanza, de Nuevo Chimbote.

\section{Tabla 4}

Prueba t student para medir la influencia de los Factores de atención y cuidado en la familia en el diagnóstico nutricional normal

95\% Intervalo de confianza para la diferencia

\begin{tabular}{|c|c|c|c|c|c|c|}
\hline & $\mathrm{t}$ & gl & $\begin{array}{c}\text { Sig. } \\
\text { (bilateral) }\end{array}$ & $\begin{array}{l}\text { Diferencia } \\
\text { de medias }\end{array}$ & Inferior & Superior \\
\hline Niños con diagnóstico normal & $-677,989$ & 46 & ,000 & $-48,872$ & $-49,02$ & $-48,73$ \\
\hline $\begin{array}{l}\text { ¿Qué hacía cuando le da o le } \\
\text { dio de lactar a su niño o niña? }\end{array}$ & $-172,360$ & 46 &, 000 & $-46,191$ & $-46,73$ & $-45,65$ \\
\hline $\begin{array}{c}\text { ¿Cómo le da de comer a su niño } \\
\text { o niña? }\end{array}$ & $-147,326$ & 41 & ,000 & $-46,238$ & $-46,87$ & $-45,60$ \\
\hline $\begin{array}{c}\text { ¿Qué hace cuando su niño o } \\
\text { niña llora? }\end{array}$ & $-310,055$ & 46 &, 000 & $-48,340$ & $-48,65$ & $-48,03$ \\
\hline $\begin{array}{c}\text { ¿Con cuánta frecuencia abraza, } \\
\text { besa y le dice a su hijo que lo } \\
\text { quiere? }\end{array}$ & $-1188,923$ & 46 & ,000 & $-48,915$ & $-49,00$ & $-48,83$ \\
\hline
\end{tabular}

De acuerdo a la prueba t student, según se observa en la tabla 4, los factores de cuidado en la familia tales como: ¿Qué hacía cuando le da o le dio de lactar a su niño o niña? ¿Cómo le da de comer a su niño o niña? ¿Qué hace cuando su niño o niña llora? ¿Con cuanta frecuencia abraza, besa y le dice a su hijo que lo quiere? influyen significativamente en el diagnóstico nutricional normal. Siendo la probabilidad Sig. (bilateral $=.000$ ) es menor que alfa $(\alpha$ $=0.05$ ) a un nivel de significancia del 95\%. Por lo tanto, se rechaza la hipótesis nula y se acepta la hipótesis principal, se Concluye que los factores de atención y cuidado de la familia influyen significativamente en el diagnóstico nutricional normal de niños de 0 a 3 años del AA.HH. Nueva Esperanza, de Nuevo Chimbote. 


\section{CONCLUSIONES}

- Los factores nutricionales influyen y son altamente significativos en el diagnóstico nutricional normal que las madres practican para mantener a sus niños nutricionalmente sanos.

- Los factores de cuidado de atención en salud influyen y son altamente significativos en el diag- nóstico nutricional normal que las madres practican para mantener a sus niños nutricionalmente sanos.

- Los factores de cuidado de la familia, influyen y son altamente significativos en el diagnóstico nutricional normal que las madres practican para mantener a sus niños nutricionalmente sanos.

\section{REFERENCIAS BIBLIOGRÁFICAS}

1. Behrman, R. (2000). Tratado de Pediatría Volumen 1. I 16ta ed McGraw Hill Interamericana México pg1351.

2. Delgado Hernán y Víctor Valverde. (1986). Manual de Antropometría física. Guatemala: Instituto de Nutrición de Centro América y Panamá.
3. OMS, (1995), El estado físico: Uso e interpretación de la Antropometría. Informe de un comité de expertos de la OMS Ginebra.: Organización Mundial de la Salud.

4. Plan Nacional de Acción por la Infancia y la Adolescencia (2002-2010) Recuperado de http:// www.oei.es/quipu/peru/PNAIA_2002_2010.pdf. 